\title{
Sparsity-Based Modelling of Compact Target Echo in PCL Radar
}

\author{
J. MisiuREWICZ* \\ Institute of Electronic Systems, Warsaw University of Technology \\ Nowowiejska 15/19, 00-665 Warsaw, Poland
}

\begin{abstract}
A passive coherent location radar makes use of transmitters already present in its environment that illuminate the surveyed area. A passive radar consists solely of a receiver that collects the waves reflected from objects of interest (targets in radar terminology) and correlates them with a direct wave from the transmitter. As the illumination is continuous, the radar suffers from near-far effect: strong echoes from near objects blind the detector from detecting weaker echoes of far objects. This may be mitigated with CLEAN method, which, however, requires precise strong echo modelling to be effective. The paper presents a method for accurate modelling of an echo in case of compact target - i.e. when signal bandwidth is insufficient for accurate resolving the target details. The method is based on the assumed sparsity of the target, which is usually adequate, as a typical object acts as a small number of point scatterers with respect to radar waves. The modelling method is analyzed with respect to the residual power after removal of modelled echo from the original signal.
\end{abstract}

PACS: $84.40 . \mathrm{Xb}$

\section{Introduction}

In a passive coherent location (PCL) radar, the target is illuminated with a broadcast signal from the transmitter of opportunity, and the radar receiver makes "parasitic" use of the transmitted signal $[1,2]$. The sounding (illuminating) signal is deterministic from the transmitter's operator point of view, but its content is not controlled from the radar side. Moreover, its properties are not shaped intentionally for the use as location signal. In the literature, it is usually assumed that the signal is random with approximately white spectrum in the transmission band [3]. Spectrum whiteness is a desired property of the illuminating signal, thus modern digital modulation schemes (e.g. defined by digital television standards) ease the PCL radar design [4].

A PCL radar operates with bistatic geometry - the transmitter and receiver are located in different points in space (see Fig. 1). Thus, the measurement of delay between the emission of the signal and reception of the echo allows us to calculate the bistatic range to the object, defined as the sum of transmitter-object and objectreceiver distances $r_{\text {to }}+r_{\text {or }}$. The delay is measured with help of the reference channel that supplies the information on the transmitted signal. Typically, the reference channel consists of an additional antenna directed towards the transmitter and connected to an additional radio receiver. In this setup, the delay between the ref-

* e-mail: jmisiure@elka.pw.edu.pl erence antenna signal and measurement antenna signal is actually proportional to $r_{\mathrm{to}}+r_{\mathrm{or}}-r_{\mathrm{tr}}$. To calculate the bistatic range, $r_{\text {tr }}$ must be known - the knowledge of geographical location of both the transmitter and receiver is necessary. The change of bistatic range is causing the observed Doppler shift in the received signal frequency. In all the following, if it is not marked otherwise, we will understand range and velocity notions as the bistatic ones.

In a PCL radar setup, the transmitter of opportunity and radar receiver are active continuously. In the result, strong echoes of near targets are present at the receiver simultaneously with weak echoes of far targets, which makes the detection of the weak echoes difficult.

A detector in a passive radar correlates the signal received from the measurement antenna with a template

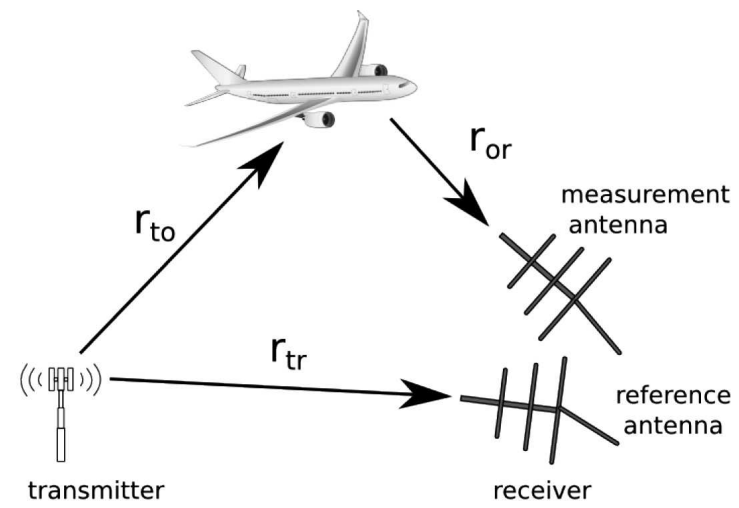

Fig. 1. Bistatic geometry of PCL radar sensing. 
constructed from the reference signal, then a thresholding procedure is applied in order to detect correlation peaks. Typically, the reference signal is captured with an antenna directed towards the transmitter, so it is disturbed with reception noise. Moreover, if the target moves, the received signal is not a copy of the transmitted signal. The Doppler effect produces carrier shift that results in a received baseband signal modulation; also, the complex envelope of the signal is contracted or dilated in time. The first effect is usually compensated for with a modulation of the template, which leads to the construction of a bank of matched filters designed for different target velocities. The second effect may be neglected unless the integration time in the processor is very long.

At the output of a correlation processor a rangeDoppler correlation function is calculated, with maxima indicating possible targets. A strong echo produces a distinct peak accompanied by significant sidelobes in the correlation function. Thus, a weaker echo may be masked by these sidelobes.

The problem of near and far target echo separation is also present in a typical active $\mathrm{CW}$ radar with linear frequency modulation, but it can be dealt with by simple filtering of the received signal, as the echoes are also separated in frequency. In passive and noise radars which use correlation receivers and noise-like wave forms, the problem is more complex.

In the past, many methods of unwanted signal removal originating from the CLEAN technique have been proposed. The CLEAN technique, introduced in 1970's for the reconstruction of blurred radioastronomy images [5], is based on subtracting the modelled echo of a point scatterer from the signal. A similar ground clutter and crosstalk signal removal procedure for a noise radar was proposed in [6] and [7].

The removal of a strong target echo with an adaptive technique and a point-like scatterer model was proposed in [8] for a passive radar.

As seen in Fig. 2, CLEAN processing consists of detecting a strong echo, estimating its parameters, modelling an ideal echo with given parameters and subtracting it from the signal. The resulting signal - with strong echo cancelled - undergoes the detection once again, to reveal weaker echoes.

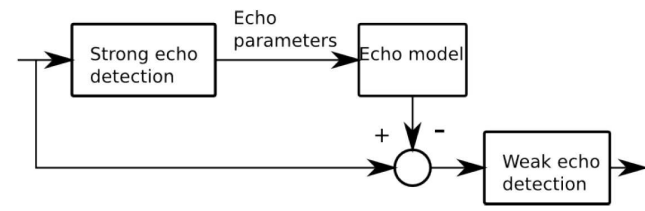

Fig. 2. The idea of CLEAN processing in PCL radar.

In order to obtain good cancellation of an echo, high accuracy of target range and the Doppler shift estimation is necessary. The experiments [9] show that the proposed procedure of successive echo removal fails in the case of two point scatterers positioned very close on the range-
-Doppler plane; they are detected as one echo, and the remainders after the removal of this echo are not modelled properly. In the effect, the improvement of the weak target detection is limited to just few $\mathrm{dB}$.

A typical man-made target viewed in radar wavelength of several $\mathrm{GHz}$ consists of a small number of dominant scatterers, formed at edges or at dihedral structures (Fig. 3). Other parts of the target form much weaker echoes which do not play a role in masking.

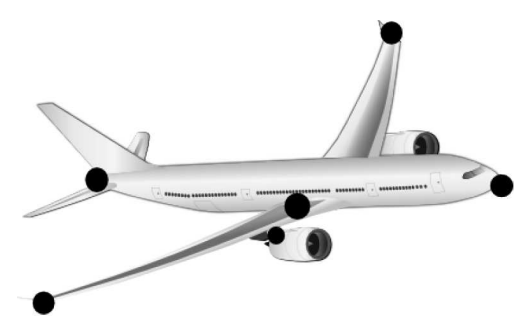

Fig. 3. Typical location of strong scatterers in a target.

In this paper, a method based on a joint modelling of a multipoint target echo is proposed. It is shown that simultaneous modelling of several point scatterers allows us to remove a complex echo more cleanly.

\section{Problem formulation}

In a PCL radar the illuminating signal is modelled as a continuous-wave signal with noise-like modulation of carrier. The bistatic range to the target is then estimated by finding a maximum of crosscorrelation between the received echo signal and a copy of the transmitted signal retrieved from the reference channel.

The crosscorrelation calculated from a finite-time segment of the signal has a form of a distinct peak and a noise floor. To fight the effect of masking weak echoes by the noise floor of strong echo, CLEAN procedure is used.

\subsection{Received signal model}

Let us express the transmitted signal in the form of a band-limited noise (or pseudo-noise) envelope $x_{\mathrm{T}}(t)$ modulating the carrier of frequency $F_{\mathrm{c}}$ :

$$
X_{\mathrm{T}}(t)=x_{\mathrm{T}}(t) \cos \left(2 \pi \mathrm{j} F_{\mathrm{c}} t\right) .
$$

The bistatic range $r$ from a radar to a moving target can be approximated with a linear function of time $r=r_{0}+v t$, where $v$ is the bistatic component of the object velocity. The received echo of a single target will be delayed by $t_{\mathrm{d}}=\frac{2 r}{c}=\frac{2 r_{0}+2 v t}{c}$. The delay will show up on the receiving side as the carrier phase change and Doppler shift, and as the envelope delay and stretch. In the following, complex value $A_{0}$ denotes the amplitude change of the received signal, incorporating all the attenuation in the propagation and reflection, and also phase change at the reflection. The $\xi(t)$ represents the additive noise in the received signal

$$
\begin{aligned}
& X_{\mathrm{R}}(t)=A_{0} X_{\mathrm{T}}\left(t-t_{\mathrm{d}}\right)+\xi(t) \\
& \quad=A_{0} x_{\mathrm{T}}\left(t-t_{\mathrm{d}}\right) \cos \left(2 \pi \mathrm{j} F_{\mathrm{c}}\left(t-t_{\mathrm{d}}\right)\right)+\xi(t) .
\end{aligned}
$$


In the demodulation process, the signal is shifted in frequency to the baseband. The demodulated signal can be represented as a complex function of time

$$
\begin{aligned}
& x_{\mathrm{R}}(t)=A_{0} x_{\mathrm{T}}\left(t-t_{\mathrm{d}}\right) \mathrm{e}^{-2 \pi \mathrm{j} F_{\mathrm{c}} t_{\mathrm{d}}} \\
& \quad=A_{0} x_{\mathrm{T}}\left(t-\frac{2 r_{0}+2 v t}{c}\right) \mathrm{e}^{-2 \pi \mathrm{j} F_{\mathrm{c}} \frac{2 r_{0}+2 v t}{c}} .
\end{aligned}
$$

For the simplicity, we drop the additive noise in the above equation.

The velocity-dependent envelope stretch $2 v t / c$ can be neglected - it is important only with long observation times and high object velocities. Finally, the signal reflected from a set of $N$ reflecting points can be expressed as

$$
x_{\mathrm{R} N}(t)=\sum_{i=1}^{N} A_{i} x_{\mathrm{T}}\left(t-\frac{2 r_{i}}{c}\right) \mathrm{e}^{-2 \pi \mathrm{j} F_{\mathrm{c}} \frac{2 v_{i} t}{c}},
$$

where we incorporate the initial phase shift $\mathrm{e}^{-2 \pi \mathrm{j} F_{\mathrm{c}} \frac{2 r_{i}}{c}}$ in a complex value of $A_{i}$.

\subsection{The receiver}

In the receiver, a matched filter for the expected echo signal is constructed from the reconstructed transmitted signal template. Such a filter with the impulse response length (integration time) of $T_{i}$ is theoretically producing the gain of $B T_{i}$ in the reception of a signal, having band width of $B$, in the presence of white noise. A filter for a Doppler shifted signal is obtained by modulating the template with the Doppler frequency. The operation of a receiver matched filter, or correlation processor is described by the following equation (envelope stretch neglected):

$$
y(r, v)=\int_{t=0}^{T_{i}} x_{\mathrm{R}}(t) x_{\mathrm{T}}^{*}\left(t-\frac{2 r}{c}\right) \mathrm{e}^{\mathrm{j} 2 \pi F_{\mathrm{c}} \frac{2 v}{c} t} \mathrm{~d} t .
$$

Local maxima of the output function $y(r, v)$ are declared as detected targets.

Usually, the processing is done by digital means, so the discrete-time equivalent of the above equation is evaluated for a discrete set of values of the delay $r_{0}$ and velocity $v$.

\subsection{Masking effect}

The object is illuminated with a continuous-wave band pass signal with band width $B$ and transmitted power $P_{\mathrm{T}}$ usually defined by relevant standards of radio frequency usage. The reflecting property of the object is usually reduced in analysis to a single figure $S$ called effective radar cross-section (RCS) expressed in $\mathrm{m}^{2}$. The received power is then described by a formula

$$
P_{\mathrm{R}}=\frac{P_{\mathrm{T}} G_{\mathrm{T}} G_{\mathrm{R}} S \lambda^{2}}{(4 \pi)^{3} r_{\mathrm{to}}^{2} r_{\mathrm{or}}^{2}},
$$

where $G_{\mathrm{T}}$ and $G_{\mathrm{R}}$ denote gains of transmit and receive antennas, $\lambda$ is the carrier wavelength, $r_{\text {to }}$ or $r_{\text {or }}$ denote the distance between transmitter and object or between object and receiver.
It can be seen from this equation that in practice the received echo is usually very weak. The ability to detect far object echo is a result of correlation processing with long integration time $T_{i}$, which enhances the signal to noise power ratio by $B T_{i}$. Due to noise-like (wide-band and non-periodic) structure of signal, this product may attain values as high as $50 \mathrm{~dB}\left(10^{5}\right.$ in linear scale). With detection threshold set at $15 \mathrm{~dB}$ over the noise level at the correlator output, the weakest detectable signal at the receiver input may be even $35 \mathrm{~dB}$ below the noise level.

The correlation receiver described by (5) is optimal in the MS sense for one point target echo present in the received signal. We will further study a more complex case, when one strong echo originating from a nearby target and one weak echo originating from a far target are present. Then, detection of the first, strong echo will be performed properly, but the output of the correlation receiver tuned to the weak echo delay and velocity will be disturbed by the strong interference caused by the first echo. As the transmitted signal has the properties of noise, the effect will be equivalent to raising the noise floor for the detection of the weaker echo. In the range-rangDoppler plane it may be also viewed as the sidelobes of the strong echo. The correlation receiver described by (5) is optimal in the MS sense for one point target echo present in the received signal. We will further study a more complex case, when one strong echo originating from a nearby target and one weak echo originating from a far target are present. Then, detection of the first, strong echo will be performed properly, but the output of the correlation receiver tuned to the weak echo delay and velocity will be disturbed by the strong interference caused by the first echo. As the transmitted signal has the properties of noise, the effect will be equivalent to raising the noise floor for the detection of the weaker echo. In the range-Doppler plane it may be also viewed as the sidelobes of the strong echo.

With raised noise floor, an effective range of detection for a weak echo is reduced. This is called masking effect in the literature [8].

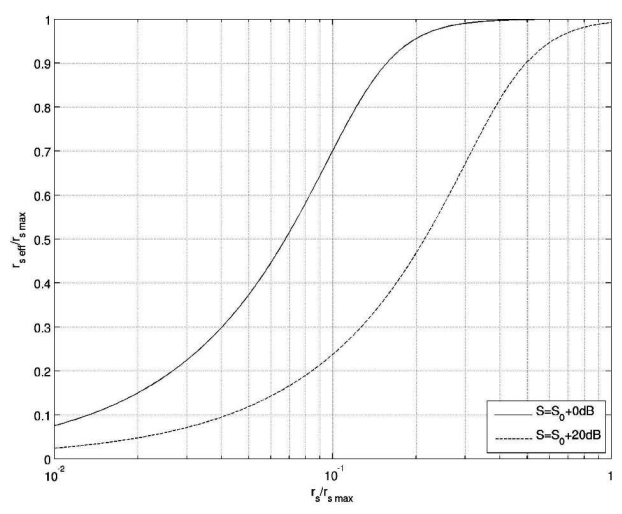

Fig. 4. Reduction of PCL radar detection range in presence of a strong unwanted echo. 
The problem is depicted in Fig. 4. In order to alleviate the problem of many independent variables, the following normalization was made. The range variable in the plot $r_{\mathrm{s}}$ is a geometric mean of $r_{\mathrm{to}}$ and $r_{\mathrm{or}}$, normalized to its maximum value $r_{\mathrm{s} \text { max }}$ still allowing the detection of an object with given radar cross-section of $S_{0}$. Thus, effective (reduced) range $r_{\text {eff }}$ could be calculated with assumption that a strong reflecting object of RCS equal to $S$ is positioned at the distance of $r_{\mathrm{s}}$. The detection threshold of $15 \mathrm{~dB}$ above noise level was assumed with integration gain $B T_{i}$ equal to $50 \mathrm{~dB}$.

\subsection{Strong echo cancellation}

If the strong echo is removed from the received signal, also the processing noise of this echo is cancelled. The basic procedure of removing the strong echo from the signal is based on the idea of CLEAN processing used in radioastronomy. First, a strong target is detected and its position $r_{1}$ and velocity $v_{1}$ are estimated from the coordinates of the maximum of $|y(r, v)|$. Then, the estimated $\hat{r_{1}}, \hat{v_{1}}$ and the amplitude $\hat{A_{1}}=y\left(\hat{r_{1}}, \hat{v_{1}}\right)$ are used for modelling the target echo. Finally, the modelled echo is subtracted from the received signal,

$$
x_{\mathrm{R}}^{(1)}(t)=x_{\mathrm{R}}(t)-\hat{A_{1}} x_{\mathrm{T}}\left(t-\frac{2 \hat{r_{1}}}{c}\right) \mathrm{e}^{-2 \pi \mathrm{j} F_{\mathrm{c}} \frac{2 \hat{v}_{1} t}{c}}
$$

and the weaker target may be then detected in the "cleaned" signal $Y$. The process may be repeated several times to detect and remove all the echoes that are strong enough to cause interference.

The effectiveness of the removal may be assessed with the residual power level after the subtraction of the echo in the case of only one echo present in the signal. It is usually desired that the residual power level be as low as -40 or even $-60 \mathrm{~dB}$ with respect to the strong echo power, so that in the real case the residual noise-like signal remains well below the thermal noise floor. Such an exact removal requires very accurate estimation of the $\hat{r_{1}}$ and $\hat{v}_{1}$ usually with the accuracy much better than one range or velocity cell.

Figure 5 shows the effectiveness of the removal with respect to the accuracy of range estimation [9]. The residual power $P_{\text {err }}$ has been normalized to the initial strong echo power $P_{0}$. In has been plotted versus the range estimation error $r_{\text {err }}$ normalized to the radar range resolution cell $\Delta_{\mathrm{r}}=c / B$, where $B$ is the illuminating signal band width and $c$ is the electromagnetic wave velocity.

In practice, a numerical minimization procedure is frequently used here to find a residual power minimum. The procedure is effective if the target can be modelled as a single reflecting point.

\subsection{Complex target}

The above removal procedures can be effective if the assumption of the point-like nature of target is true and the strong scattering points are well isolated. If, however, two or more scattering points of similar amplitude are positioned close together (they are not resolvable due to low

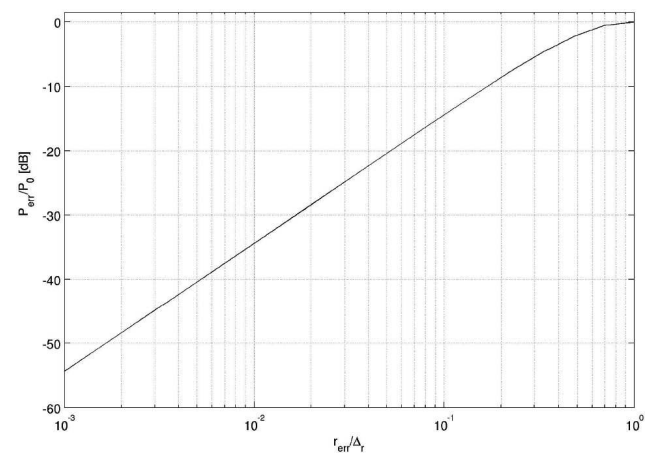

Fig. 5. Residual power after cancelling strong echo with poor range estimate.

band width of the sounding signal, although they are well separated with respect to carrier wavelength) and their echoes interfere in the sampled signal in a non-orthogonal way (i.e. in the distance comparable to a radar resolution cell). Both the $|y(r, v)|$ maximum search and the residual power minimum search procedures usually find a point "between" these targets on the range-Doppler plane. The removal of such an echo is much less effective, and the residuals also do not fit well into the point echo model so the successive removal iterations do not "clean" them well. This happens when target details are closer together than the velocity of light times reciprocal of bandwidth, $\Delta r<c / B$. Such a target will be called "compact" in the sequel.

With sufficient band width, a noise-like correlation structure of the signal would permit separate modelling of point reflectors, as their contributions would be quasi-orthogonal. In the insufficient band-width case the compact, complex echo components must be modelled in a joint approach.

\subsection{Target model}

We assume that the target consists of a small number of point-like reflectors located (in the bistatic range dimension) within one resolution cell, i.e. closer to each other than $c / B$, where $B$ is the sounding signal band width and $c$ is the velocity of the electromagnetic wave. Thus, the target impulse response $h(n)$ is modelled as a series of $A_{k} \delta\left(t-t_{k}\right)$ components,

$$
h(t)=\sum_{i=1}^{K} A_{k} \delta\left(t-t_{k}\right),
$$

where $A_{k}$ is a complex reflection factor (describing amplitude and phase of the reflection from $i$-th point reflector) and $t_{k}$ is the bistatic delay associated with the reflector position.

Signal processing in a PCL radar usually takes place in the base band, after demodulation from the radio band. In the following we will consider base-band representation of relevant signals. To simplify the analysis we will also leave out the problem of the Doppler shift in the received signal. 
The impulse response of the base-band representation of a target will include in $A_{k}$ factors the phase component associated with reflection, together with the phase factor associated with delay of the carrier wave by $t_{k}$. The received echo of $K$-point scatterer can be described as

$$
\begin{aligned}
y(t) & =\sum_{k=1}^{K} A_{k} x\left(t-t_{k}\right)=x(t) * h(t) \\
& =x(t) * \sum_{k=1}^{K} A_{k} \delta\left(t-t_{k}\right),
\end{aligned}
$$

where $x(t)$ and $y(t)$ are complex base-band representations of the transmitted and received signals respectively, $h(t)$ represents the impulse response of the target, and * is a convolution operator. It must be noted that in a PCL receiver the $x(t)$ signal is practically obtained by radio reception from the reference antenna, so it is known with accuracy limited by the addition of some noise.

The relation (9) might be rewritten in the frequency domain as

$$
Y(\omega)=X(\omega) \sum_{k=1}^{K} A_{k} \mathrm{e}^{-\mathrm{j} \omega t_{k}}=X(\omega) H(\omega) .
$$

If we model $X(\omega)$ as a realization of white noise filtered to the band width of $\left(-\omega_{\mathrm{g}},+\omega_{\mathrm{g}}\right)$ where $\omega_{\mathrm{g}}=2 \pi B / 2$ we can see that $Y(\omega)$ contains only the low-pass part of the information about $A_{k}$ and $t_{k}$. We cannot restore the components of $h(t)$ exactly, due to noise in measured signals. Thus the goal of the modelling process is to find, without engaging too much computational power, a relatively simple model that allows to reconstruct a signal $\hat{h}(t)$ that is a good approximation of $h(t)$ in the band width of interest $\left(-\omega_{\mathrm{g}},+\omega_{\mathrm{g}}\right)$. If we take the error power as the quality measure, we may calculate it in the spectral domain as

$$
P_{\text {err }}=\int_{-\omega_{\mathrm{g}}}^{\omega_{\mathrm{g}}}|h(\omega)-\hat{h}(\omega)|^{2} \mathrm{~d} \omega .
$$

\subsection{Model reconstruction problem}

The problem of finding the target model - i.e. determining the values of $A_{k}$ and $t_{k}(k=0 \ldots K)$ in (9) - is nonlinear with respect to $r_{k}$, moreover the number $K$ is unknown. It may be solved with a nonparametric method - by discretizing the frequency variable $\omega$ in the interval $\left(-\omega_{\mathrm{g}},+\omega_{\mathrm{g}}\right)$ and assuming that delays $t_{k}$ belong to a set of $M$ discrete points $t_{m}$ uniformly sampling the interval reflecting the expected object spread. The delay discretization step size should respect the required accuracy of $t_{k}$ estimation.

Knowing $Y(\omega)$ and $X(\omega)$ at the discrete $\omega$ values one may construct a system of linear equations with unknowns $A_{m}$ corresponding to the reflectivity values at assumed discrete $t_{m}$ delays. This system can be solved in the mean squares (MS) sense, and the solution minimizes MS error in discrete frequency points.
This approach has, however, some important drawbacks. First, in order to obtain good strong echo removal, the number $M$ of delay discretization points should be from 100 to about 1000 (see Fig. 5). Second, the system may be ill-conditioned due to strong dependence between succesive samples in delay domain. The solution will be, in effect, very sensitive to noise in the signal as well as to other echoes out of the considered range interval. Finally, the MS solution need not reflect the desired model — usually most $A_{m}$ will have significant magnitudes.

Another approach is a parametric one. Assuming small number $K$ of components one may find $A_{k}$ and $r_{k}$ by minimizing error using numerical optimisation methods. Also, usage of methods from the domain of frequency estimation may be considered (e.g. MUSIC type methods).

With parametric approach the key element is to properly assume the number of components $K$. Each component is represented by 2 parameters (one of them complex) which contribute to the computational complexity. The complexity increases again when target velocity is added to the parameter set.

It has been shown in [9] that usually up to 3 points in the model suffice to achieve desired removal quality this is a result of strong dependence between responses of closely spaced reflectors.

This observation leads to the application of sparsity-based methods. It is known [10] that many inverse problems unresolved with band-limited signal model may be resolvable with assumption of sparse signal model. Recent developments in the convex optimization programming $[11,12]$ have provided efficient ways of solving sparse reconstruction problems using fast numerical algorithms.

\subsection{Reconstruction based on sparsity assumption}

Sparsity of signal model is defined as the ability to represent the signal $y(t)$ with a linear combination of $M$ basis vectors $\psi_{m}(t)$ :

$$
y(t)=\sum_{m=1}^{M} A_{m} \psi_{m}(t),
$$

where the number of non-zero $A_{m}$ coefficients (usually denoted by $\|A\|_{0}$, however it must be underlined that $\|\cdot\|_{0}$ does not have all properties of a norm) is much lower than $M$. The set $\left\{\psi_{m}\right\}$ is sometimes called a dictionary.

The reconstruction problem is then posed as finding the unknown $A_{m}$ coefficients from known samples of $y(t)$, taken at known instants $t_{n}$. In the following we will denote by $y$ the column vector of $y\left(t_{n}\right)$. We will also use the symbol of $P$ to denote a matrix constructed with all the $\psi_{m}\left(t_{n}\right)$ as the consecutive rows. Thus, our knowledge about the signal can be formulated as

$$
y=P a,
$$

where $a$ is the column vector of unknown $A_{m}$ values.

Using the knowledge that a signal is sparse, it is possible to reconstruct $a$ with much lower number of time samples in $\left\{t_{n}\right\}$ than dictated by the Nyquist theorem. 
Contrary to well-known parametric methods of reconstructing linear combinations of signals with known models (e.g. MUSIC) it is not necessary to know the $\|a\|_{0}$ value a priori.

It should be, however, noted that not all choices of sampling instants allow the reconstruction [10].

The methods used to reconstruct sparse signals are based on searching for $\tilde{a}$ that minimizes the $\ell_{1}$ norm $\|\tilde{a}\|_{1}=\sum_{m}\left|\tilde{a}_{m}\right|$ and fulfills the condition of Eq. (13). The usage of $\ell_{1}$ norm to promote low sparsity value $\|\tilde{a}\|_{0}$ has been adopted in many sparse reconstruction techniques [13], as direct minimization of $\|\tilde{a}\|_{0}$ is an nondeterministic polynomial-time hard (NP-hard) problem.

In the passive radar application, the dictionary of $\psi_{m}$ vectors is equal to the set of the template signals $x\left(t-\tau_{m}\right)$ with discretized delays $\tau_{m}$.

The reconstruction problem in PCL has to be solved in the presence of noise. Remembering that the main purpose of model reconstruction is the cancellation of strong echo, we will understand that the noise component models weaker echoes (uncorrelated with the strong one) as well as ordinary receiver noise.

The presence of noise makes the exact fulfillment of Eq. (13) practically impossible. However, the noise may be assumed uncorrelated with the dictionary components. In conclusion, the exact equality should be replaced by approximate equality with sufficiently low MS error.

In the sparse reconstruction community the (13) problem relaxation in the presence of noise is called least absolute selection and shrinkage operator (LASSO). First variant of this problem (a "constrained norm" variant) is formulated as follows [10]:

$$
\min \|\tilde{a}\|_{1} \text { subject to }\|P \tilde{a}-y\|_{2} \leq \varepsilon .
$$

A second variant (a "scaled norm" one, also called "basis pursuit denoising") is formulated as

$$
\min \left(\|P \tilde{a}-y\|_{2}^{2}+\lambda\|\tilde{a}\|_{1}\right),
$$

where $\lambda$ parameter accounts for balancing between reconstruction accuracy and sparsity.

In PCL radar the power of noise component is not known a priori, so it is more justified to use the "scaled norm" variant of posing the problem.

\section{Experimental results}

In order to confirm the ideas presented in the above, a series of experiments has been performed. A received signal has been simulated with a known compact echo model, using sampling frequency 200 times higher than the band width of sounding signal. With this oversampling, it was possible to simulate the fine-grained structure of the reflecting target. Next, the signal has been downsampled to reflect typical sampling conditions in a radar, with the sampling frequency approximately equal to doubled band width.
Then, the structure of the target echo has been reconstructed with several methods and the quality of reconstruction has been assessed by comparing the estimated $\tilde{a}$ to the known value $a$ and also by calculating power remaining after removing the reconstructed echo from the original signal (according to the CLEAN method). With the ideal reconstruction, the remaining power would be equal to the power of background noise used in simulating the signal.

First method of reconstruction, used as a starting point, was a least squares method with assumption of one reflecting point located on the original (coarse) range sampling grid, i.e. with only $a_{0}$ as a nonzero coefficient in $(13)$.

Another method used as reference was a full least square (LS) approximation, finding $\tilde{a}$ value that minimizes $\|P \tilde{a}-y\|_{2}$. This is a parametric method and number of discretization steps $M$ was chosen equal to 20 .

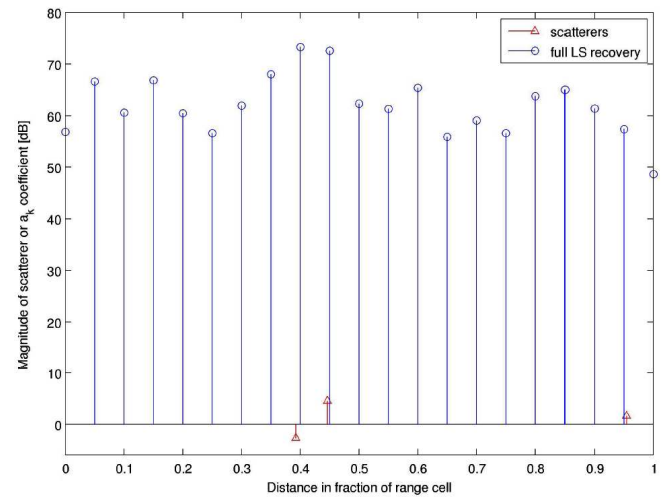

Fig. 6. The $a_{k}$ sequence recovered with full LS solution (absolute values plotted).

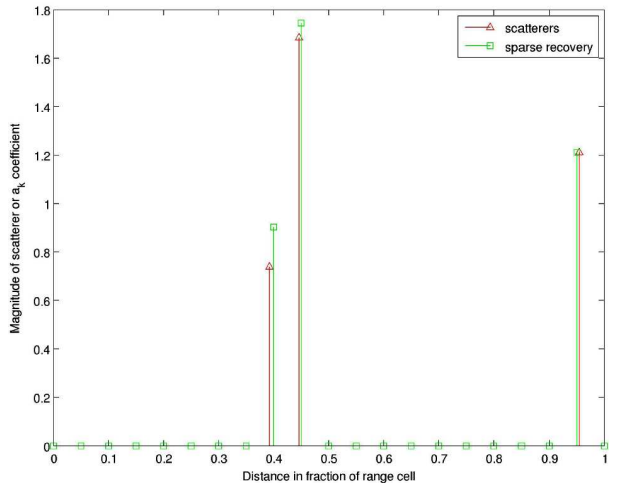

Fig. 7. The $a_{k}$ sequence recovered with sparse search (absolute values plotted).

Finally, a sparse solution has been sought. Due to numerical implementation problems, convex optimization has not been used (this is planned for further research). Instead, solutions with increasing sparsity value $\|\tilde{a}\|_{0}$ (starting from one) have been found, corresponding $\|\tilde{a}\|_{1}$ 


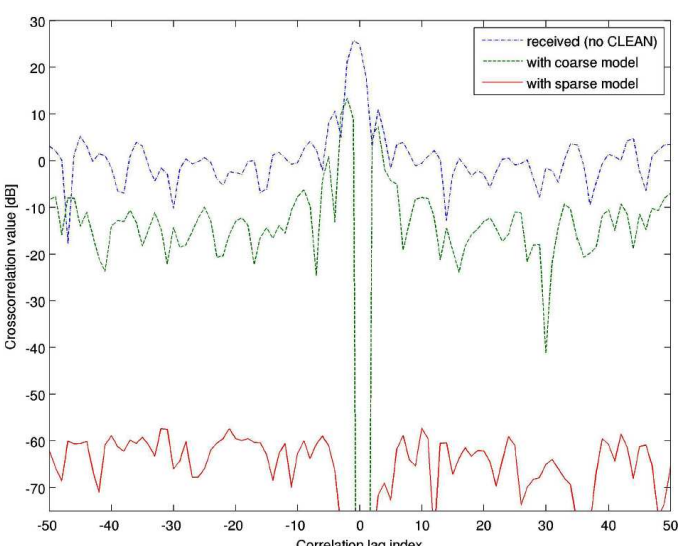

Fig. 8. Matched filter output after CLEAN (sparse model recovery), 3 scatterers, sparsity $=3$.

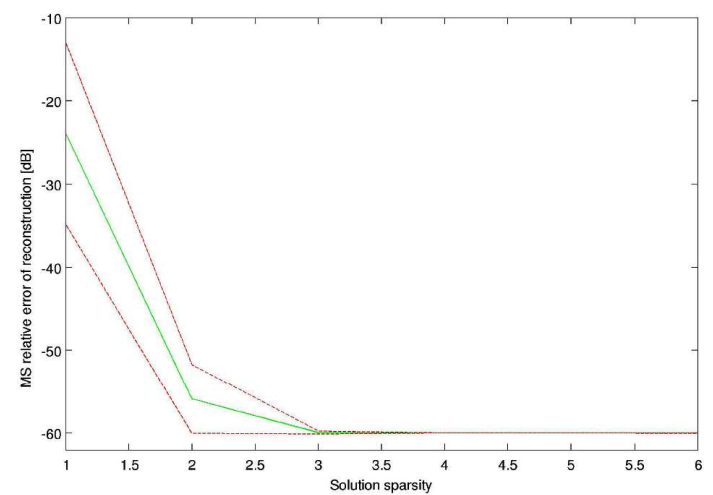

Fig. 9. Error power (Monte Carlo mean and standard deviation limits), target sparsity $=2$.

and reconstruction MS errors evaluated and plotted. The search was also based on $M=20$, i.e. the modelling template set had 20 elements.

It must be underlined that the simulated locations of reflectors were not chosen on the discretization grid. Background noise level was set to $-60 \mathrm{~dB}$ with respect to the total power of the target echo.

An example of recovery results is shown in Fig. 6 (recovery with full LS approximation) and Fig. 7 (recovery with sparse model), where absolute values of complex $a_{m}$ coefficients are plotted. Simulated number of reflectors is 3, their positions and strengths are marked with triangles. For LS method, the vertical axis is presented in logarithmic (decibel) scale to show values differing by orders of magnitude in one plot.

Results of applying CLEAN procedure with recovered echo model are shown in Fig. 8. The magnitude of the output signal from a matched filter applied to the CLEAN'ed signal is plotted. For the reference, also the output for the original received signal (before the CLEAN) and the output with coarse modelling of the echo (with scatterers modelled on range cell grid) is shown.

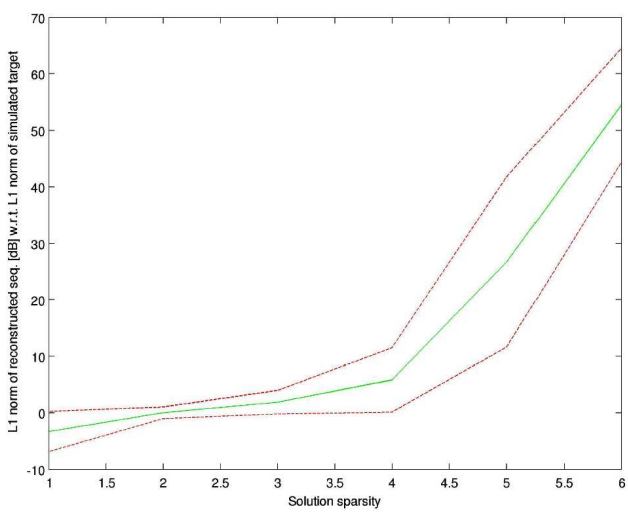

Fig. 10. The $\ell_{1}$ norm of $a_{m}$ sequence (target sparsity $=2)$.

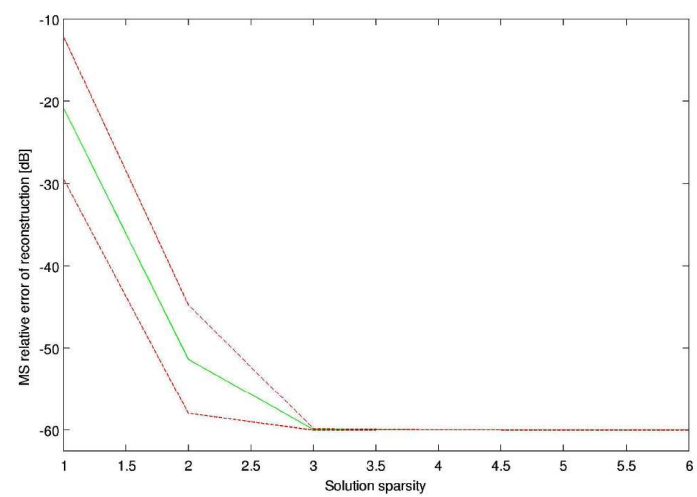

Fig. 11. Error power (Monte Carlo mean and standard deviation limits), target sparsity $=3$.

First, the coarse method gives just about $10 \mathrm{~dB}$ of improvement in the CLEAN procedure. Only the zeroth lag of crosscorrelation is cancelled completely. Clearly, fine-grained modelling is necessary to obtain better results.

Next, it is clear that the LS method is inefficient the recovered model fits the data, so the CLEAN results

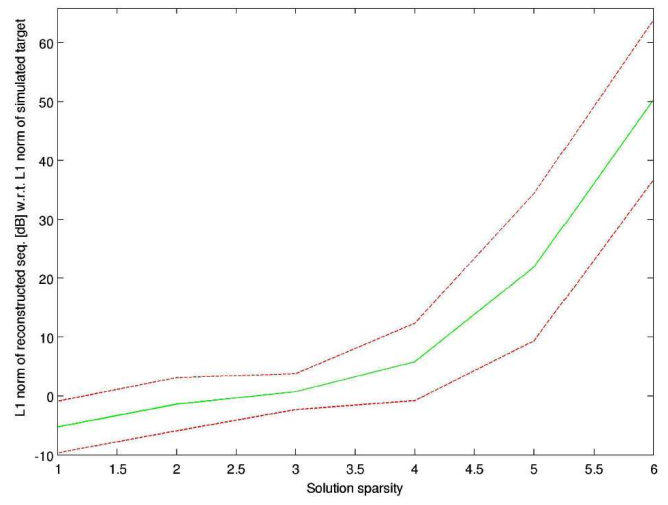

Fig. 12. The $\ell_{1}$ norm of $a_{m}$ sequence (target sparsity $=3$ ). 


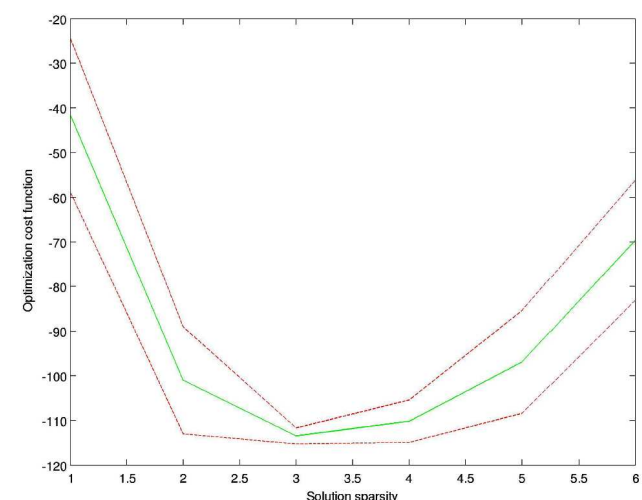

Fig. 13. "Scaled norm" cost function, target sparsity $=3$.

can be good, but the $\tilde{a}_{m}$ values are huge and they do not reflect the simulated model. With obvious lack of orthogonality between template matrix columns, the LS solution was very sensitive to the added noise.

Sparse solution, on the other hand, gives good results with CLEAN with good approximation of the echo model. It may be noted that the approximation quality increases when the number of non-zero $\tilde{a_{m}}$ values $\left(\|\tilde{a}\|_{0}\right)$ approaches the actual number of scatterers in target. The noise level limits the improvement at $-60 \mathrm{~dB}$, and with higher $\|\tilde{a}\|_{0}$ also the absolute values of $\tilde{a}_{m}$ rise quickly.

Monte Carlo simulation results are shown in next figures. Targets with different number of randomly located scatterers were simulated. The results of approximating the signal with sparse model are shown. In each pair of figures the number of scatterers is constant, and results of 30 experiments are averaged. The approximation power (Fig. 9 and Fig. 11) and $\|\tilde{a}\|_{1}$ norm of the approximation coefficients (Fig. 10 and Fig. 12) is plotted versus the number of scatterers in the model. Again, the approximation quality improves with $\|\tilde{a}\|_{0}$ increase, but $\|\tilde{a}\|_{1}$ rises sharply when the model order is higher than the actual number of scatterers.

When we compare the behaviour of MS error of modelling and $\ell_{1}$ norm of the $a_{m}$ sequence, we can see that a cost function can be defined combining these norms to obtain a minimum at the solution sparsity equal to the actual number of strong scatterers. This is equivalent to the "scaled norm" problem (see Eq. (15)). Such a cost function with $\lambda$ parameter set to $10^{-4}$ is shown for the case of 3 scatterers (Fig. 13). Minimizaton of this cost function would lead to solutions that approximate the actual model well.

\section{Conclusions}

The idea of sparse reconstruction of compact target model in a PCL radar has been presented and verified in simulations.

The results of simulations show that sparse modelling of the strong target leads to good result of applying CLEAN procedure in order to detect weaker targets.

Application of the presented results requires the implementation of the recovery process with effective methods for minimizing simultaneously the modelling error and $\ell_{1}$ norm of model coefficients. This is the area of convex optimization methods, which are witnessing a breakthrough in a wide variety of topics, and their application to the presented task may enable real-time processing with model recovery.

\section{References}

[1] P.E. Howland, in: Int. Conf. on Radar, Soc Electr. \& Electron., Paris 1994, p. 251.

[2] K.S. Kulpa, IEE Proc. Radar, Sonar, Navig. 152, 169 (2005).

[3] M. Malanowski, G. Mazurek, K.S. Kulpa, J. Misiurewicz, in: Proc. Int. Radar Symp., IRS-200\%, Cologne (Germany), German Institute of Navigation (DGON), Bonn 2007, p. 431.

[4] M. Malanowski, K.S. Kulpa, J. Misiurewicz, P. Samczyński, in: Electronic Devices and Systems - Selected Problems, Wojskowa Akademia Techniczna, Warszawa 2009 (in Polish).

[5] J.A. Högbom, Astron. Astrophys. Supp. 15, 417 (1974).

[6] S.R.J. Axelsson, in: Proc. of URSI 2005 Commission F Symp. on Microwave Remote Sensing of the Earth, Oceans, Ice, and Atmosphere, Barza d'Ispra (Italy), 2005.

[7] K.S. Kulpa, Z. Czekała, in: Proc. Int. Conf. RADAR 2004, Toulouse (France), Soc Electr. \& Electron, Toulouse 2004, p. 236.

[8] K.S. Kulpa, Z. Czekała, IEE Proc. Radar, Sonar, Navig. 152, 174 (2005).

[9] J. Misiurewicz, Elektronika - konstrukcje, technologie, zastosowania 8, 30 (2009) (in Polish).

[10] E.J. Candes, M.B. Wakin, Sign. Proc. Mag. IEEE 25 21 (2008).

[11] J. Mattingley, S. Boyd, Sign. Proc. Mag. IEEE 27, 50 (2010).

[12] M. Zibulevsky, M. Elad, Sign. Proc. Mag. IEEE 27, 76 (2010).

[13] J. Romberg, Sign. Proc. Mag. IEEE 25, 14 (2008). 